\title{
Dreadful Complication of Uncontrolled Diabetes Mellitus- A Case Report
}

\author{
Dr.Pooja Jain *,Dr.Ajas Gogri $\uparrow$,Dr. Hemant R Umarji $\$$,Dr.Sonali Kadam $\S$ \\ * Assistant Professor, Department Of Oral Medicine And Radiology, Govt Dental College And Hospital, \\ Aurangabad \\ 广, Assistant Professor Department Of Oral Medicine And Radiology, Govt Dental College And Hospital, \\ Mumbai \\ $\$$ Head Of Department, Department Of Oral Medicine And Radiology, Govt Dental College And Hospital, \\ Mumbai \\ §Associate Professor, Department Of Oral Medicine And Radiology, Govt Dental College And Hospital, \\ Mumbai
}

\begin{abstract}
Osteomyelitis of the jaws was once a common and dreadful disease because of its long course and associated agony to the patient, but with advent of dental care and use of antibiotics along with improved nutrition and health education, , incidence of osteomyelitis of facial bones has decreased. Osteomyelitis of maxilla is less prevalent as compared to mandible as a result of abundant blood supply \& porous bone structure of maxilla leaving it less vulnerable to chronic infection. The uncontrolled diabetes, a polygenic disorder, is a quick growing epidemic in India. This sickness if not managed before extraction can lead to unpleasant consequences of osteomyelitis. The patient's blood sugar level ought to be in controlled state before extraction. This case report presents clinical features of a fifty three year old male patient who had undergone extraction in an uncontrolled diabetic state and later on developed osteomyelitis of maxilla. This case report also highlights the CBCT features of the same.
\end{abstract}

Keywords : Osteomyelitis, Maxilla, Necrosis, CBCT

\section{Introduction}

Osteomyelitis is an inflammation of the medulla and haversian system which eventually spreads to cortical bone and periosteum. The infection slowly establishes in bone and obstructs blood supply, which leads to necrosis of bone ${ }^{[1]}$. Osteomyelitis of jaws is caused primarily by odontogenic infection. It may conjointly arise as a complication of dental extractions, surgery, trauma, inadequate treatment of a fracture, and irradiation of jaws ${ }^{[2]}$. Systemic diseases which alter host defense like uncontrolled diabetes, severe anemia, malnutrition, sickle cell disease can be associated with osteomyelitis ${ }^{[1]}$. The common age of presentation is in the fifties to the sixties, and males are affected more than females. The affected site is usually the posterior body of mandible ${ }^{[3]}$

Diabetes mellitus is a genetically heterogenous metabolic disease, which presents with hyperglycemia and risk of microvasular damage. Uncontrolled diabetes affects all the tissues of the body, and is associated with complications of multiple organ systems ${ }^{[4]}$. In 2011 there were 366 million people with diabetes globally, and this is expected to rise to 552 million by $2030^{[5]}$. A dental practitioner should take a thorough case history and carry out all the necessary investigations especially in case of diabetes mellitus before performing any invasive procedure because failure to do so may imperil the bone health and cause undesirable complications ${ }^{[6]}$.

The first case of maxillary osteomyelitis was described by Rees in $1847^{[7]}$. According to Hudson et al and Bevin et al, maxillary osteomyelitis is less common as compared to mandibular osteomyelitis due to its collateral blood supply moreover maxillary osteomyelitis due to systemic disease such as diabetes is a rare finding ${ }^{[8,9]}$

Several reports have mentioned that osteomyelitis will be treated with success by a combination of antimicrobial therapy with surgery, either sequestrectomy or decortication of diseased bone given that systemic factors should be under control. The aim of surgery is to eliminate all of the infected and necrosed bony tissue ${ }^{[10]}$.The basic aim of this article is to present such a rare case of widespread maxillary osteomyelitis complicated by uncontrolled diabetes and to emphasize its clinical and radiographic features.

\section{Case report}

A 53 years old male patient reported to the outpatient department with the chief complaint of pain and swelling in the upper jaw region. Detailed case history revealed that patient had undergone total extraction of maxillary teeth 2 months back due to mobility, but the patient's complaint of pain was not resolved for which he reported to our institute. Patient also gave history of having diabetes and hypertension since 5 years for which 
he was on antihypertensive and oral hypoglycemic drugs. Extraoral examination showed mild diffuse swelling in bilateral cheek region which was difficult to comment on, nasolabial folds appeared normal. [Fig 1] Intraoral examination revealed exposed alveolar bone (bare bone devoid of mucosa) from 16 to 26 region. The exposed bone appeared yellowish and necrotic and on probing, there was no active oozing. Complete obliteration of buccal vestibule, and palatally swelling was seen to extend from incisors to molars bilaterally. Pus discharge was evident from one extraction socket in anterior region. On palpation the exposed maxillary alveolar fragment appeared to be slightly mobile. [fig 2,3] The patient was then subjected to radiographic investigations which included maxillary topographic occlusal view, orthopantomograph, and PA Water's view to assess the extent of necrosed maxilla. Patient was also investigated for blood sugar level which were found to be $390 \mathrm{mg} / \mathrm{dl}$.

Maxillary topographic occlusal view showed extraction sockets throughout maxilla, and floor of maxillary sinus were not traceable bilaterally. [fig 4] OPG also showed multiple extraction sockets extending from 17 till 27. The sockets showed sharp crestal edges with no evidence of lamina dura. Floor of maxillary sinus was found missing on both the sides. Enostosis was seen in periapical region of mandibular first premolar. [Fig 5]. Water's view showed near total opacification of right and left maxillary sinus with air space seen in superior portion of both maxillary sinuses. [fig 6] Patient was then subjected to CBCT examination as conventional imaging could not delineate the exact extent of bone necrosis.

\section{CBCT Findings:}

Coronal section showed severe osteolysis of maxillary alveolus and hard palate with perforation of nasal floor and floor of maxillary sinus on both sides. Near total opacification of maxillary sinuses and opacification of nasal floor with involvement of right inferior nasal concha was seen. Opacification of few ethmoid cells and mild mucosal thickening of sphenoid sinus was also seen. Maxillary alveolus appeared to be sequestrating out from the remaining maxillary bone. [fig 7]Axial section showed irregular bone loss of entire maxillary alveolus with perforation of buccal and palatal cortex at multiple areas. Right and left maxillary sinus showed discontinuity of anterior, medial and lateral walls with near total opacification of sinus cavity. [fig 8 , 9]Sagittal section showed complete destruction of floor of right and left maxillary sinuses. Discontinuity of posterior walls of maxillary sinus was seen in few areas. Mucosal thickening of frontal and sphenoidal sinuses was noted, Spheno-ethmoidal recess was patent on both sides. [fig 10, 11] On the basis of clinical and radiographic findings the provisional diagnosis of condition chronic osteomyelitis was made. The pus discharge in the localized region may be due to secondary bacterial infection on the exposed bone, hence a final diagnosis of chronic osteomyelitis, secondary to uncontrolled diabetes mellitus was made.

Patient was referred to a tertiary care centre where patient was hospitalized, diabetes control and complete surgical management (sequestrectomy and debridement) was done. Patient was recalled few months after the surgery to evaluate the surgical outcome as well for dental rehabilitation.

\section{Discussion}

In the current era of advanced medical aids, osteomyelitis presents as a rare chronic condition and is more often related to debilitated, immunosuppressed or medically compromised patients and the pattern of events will cause a diagnostic dilemma ${ }^{[11]}$. The preoperative assessment of the conditions compromising host defense like diabetes is the primary responsibility of the dental practitioner and a lot of the complications may be prevented by a thorough preoperative analysis and strict management of blood sugar levels before any invasive procedure ${ }^{[6]}$.The hyperglycemic state makes the patient susceptible to impaired wound healing hence odontogenic infection, infection of antrum or injury due to dental treatment in such patients can cause maxillary necrosis ${ }^{[12]}$. Wound healing is compromised in diabetics because of reticence of inflammatory response due to diminished chemotaxis, phagocytosis, and reduced life span of leukocytes as well as decreased tissue perfusion. Diabetic macro- and microangiopathy causes ischemia and bone necrosis ${ }^{[13]}$. After extensive perusal of literature we found that chronic osteomyelitis of maxilla in medically compromised patients like uncontrolled diabetics is a rare finding.

This fact can be attributed to better drug options available to control the blood sugar levels as well to minimize the chances of infection. In our case postoperative complication of patient is suggestive of dental unawareness as multiple extractions being performed in an uncontrolled diabetic state lead to necrosis of maxilla. The present case illustrates the importance of maintaining normal blood sugar levels in diabetic patient and also preventing infection before and after extraction in order to avoid the dreadful complications of osteomyelitis. The interesting finding was complete involvement of maxillary alveolar segment and extension on to palatal bone. Diagnosis was made on the basis of clinical and radiographic findings. Pus discharge from the localized area was considered secondary infection on the exposed bone.

Treating osteomyelitis involves non-invasive conservative, as well as invasive methods. In this case CBCT showed extensive necrosis of maxilla indicating avascular nature of diseased bone, so conservative approach with antibiotics was not sufficient ${ }^{[14,15]}$. Patient was referred to a tertiary care centre where radical 
resection of diseased maxillary bone was done, along with the management of maxillary sinus pathology using nasal endoscope ${ }^{[16]}$.

A temporary prosthesis was fabricated to deal with the oronasal communication to facilitate food intake without nasal regurgitation and also to facilitate speech. Patient had an uneventful healing, after complete healing a maxillary complete denture was constructed for the patient. No recurrence is seen in one year of follow-up period as observed clinically and radiographically. [fig 12,13,14]

\section{Conclusion}

Incidence of diabetes is rising in India, there are 50 million diabetics in India according to international diabetes federation. With increase in number of diabetics the dreadful multiorgan system complications are also increasing. As a dental clinician it is our duty to identify the signs of diabetes present in oral cavity, as we say oral cavity is a mirror to systemic health, and a proper management of hyperglycemic state should be done before proceeding with invasive procedures. With adequate management of diabetes, preoperatively, osteomyelitis and eventually loss of jaw bone can be prevented.

\section{References}

[1]. Topazian, Richard G., Morton H. Goldberg, and James R. Hupp, eds. Oral and maxillofacial infections. Saunders, 2002.

[2]. Yeoh, S. C., S. MacMahon, and M. Schifter. "Chronic suppurative osteomyelitis of the mandible: case report." Australian dental journal 50.3 (2005): 200-203.

[3]. Koorbusch GF, Fotos P, Goll KT. Retrospective assessment of osteomyelitis: Etiology, demographics, risk factors, and management in 35 cases. Oral Surg Oral Med Oral Pathol. 1992;74:149-154.

[4]. Burket, Lester William, et al. Burket's oral medicine. PMPH-USA, 2008.

[5]. Whiting DR, Guariguata L, Weil C, Shaw J (2011) IDF diabetes atlas: global estimates of the prevalence of diabetes for 2011 and 2030. Diabetes Res Clin

[6]. Pract 94: 311-321.

[7]. Shetty, Laxmi, et al. "Maxillary Osteomyelitis with Candidiasis due to Extraction in Uncontrolled Diabetes State-A Case Report." Dentistry 5.279 (2015): 2161-1122.

[8]. S P Lumba, A Nirola, B S Grewal. Healed osteomyelitis of Maxilla with tooth in the floor of nose. The Journal of Laryngology \& Otology. August 1971;85[08]:877-9.

[9]. Hudson JW (1993) Osteomyelitis of Jaws: a 50-year perspective. J Oral MaxillofacSurg 51:1294-1301.

[10]. Bevin CR, Inwards CY and Keller EE. Surgical management of primary chronic osteomyelitis: a long-term retrospective analysis. J Oral Max Fac Surg 2008; 66: 2073-85.

[11]. Aitasalo K, Niinikoski J, Grenman R, Virolainen E. A modified protocol for early treatment of osteomyelitis and osteoradionecrosis of the mandible. Head Neck 1998;20:411-417.

[12]. Manimaran K, Suresh Kannan P, Kannan R. Osteomyelitis of maxilla bilateral involvement A case report. JIADS.April-June 2011;2[2]:57-8.

[13]. Macbeth R (1952) Osteomyelitis of the maxilla. J Laryng OtolM 66: 1369-1370.

[14]. Goodson WH 3rd, Hunt TK (1979) Wound healing and the diabetic patient. Surg Gynecol Obstet 149: 600-608.

[15]. Prasad K C, Prasad S C, Mouli N, Agarwal S. Osteomyelitis in the head and neck. Acta Otolaryngol.2007;127:194-205

[16]. Arunkumar, J. S., et al. "Role of nasal endoscopy in chronic osteomyelitis of maxilla and zygoma: a case report." Case reports in medicine 2011 (2011).

1. Extra Oral picture showing mild diffuse swelling bilaterally in maxillary region.

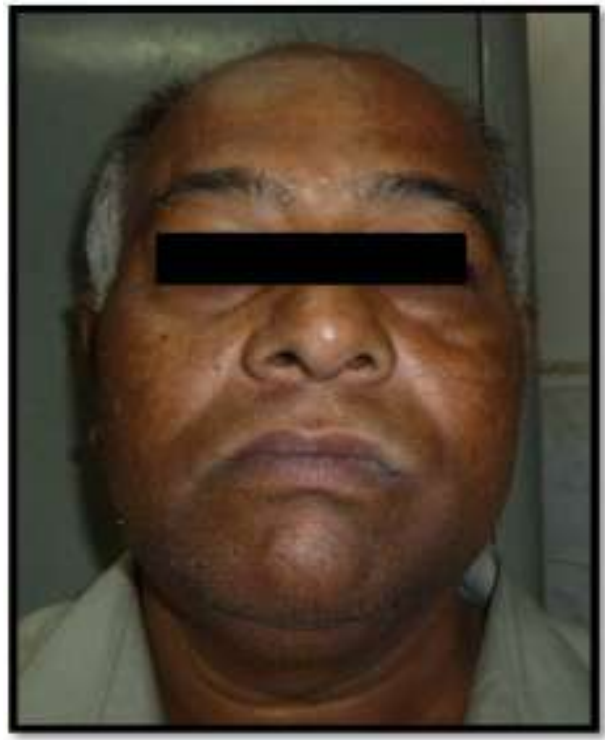

2. Intra oral picture showing exposed maxillary alveolar bone from 16 to 26 region. Note the palatal swelling also extending from molar to molar region. 


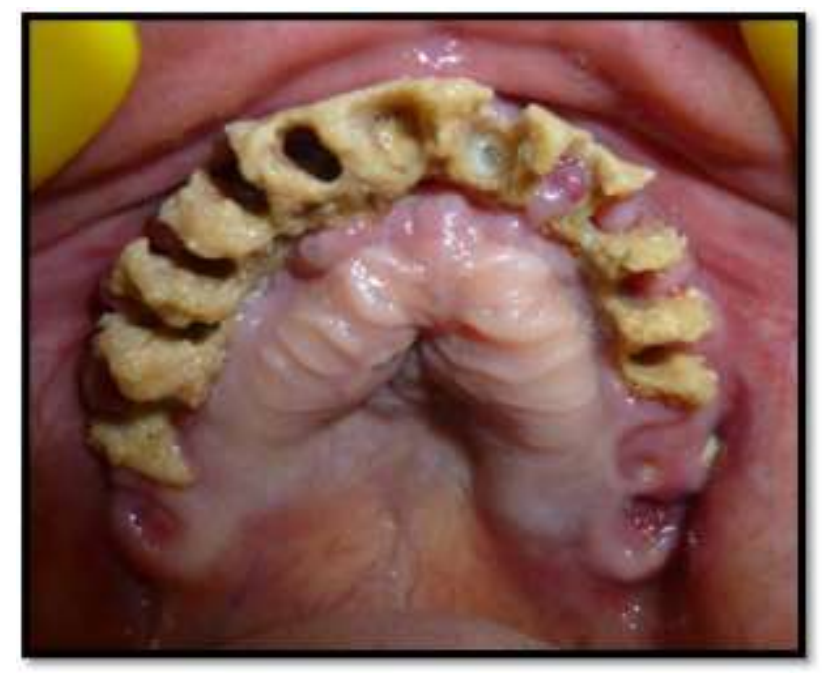

3. Close view of exposed alveolus showing denudation of overlying mucosa

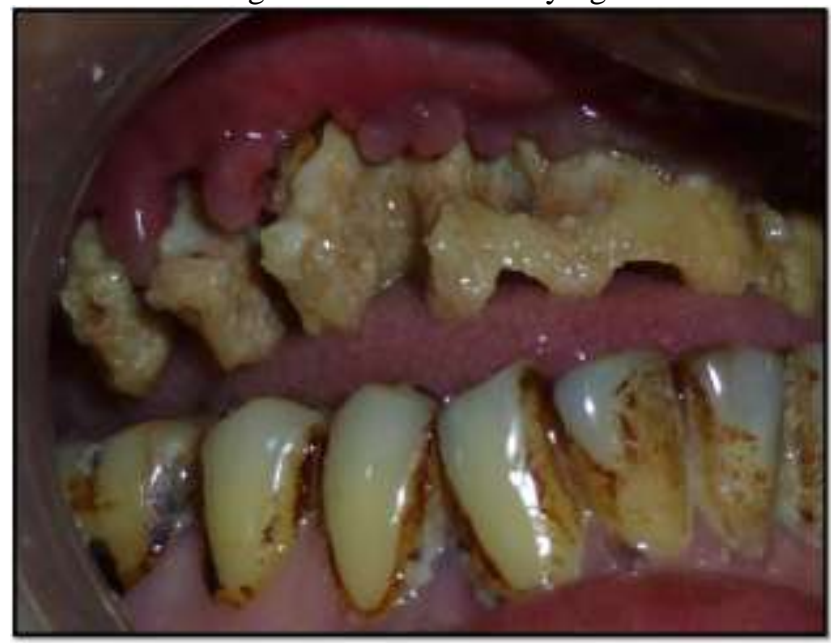

4. Occlusal view showing extraction sockets with sharp bony margins in entire maxillary alveolus.

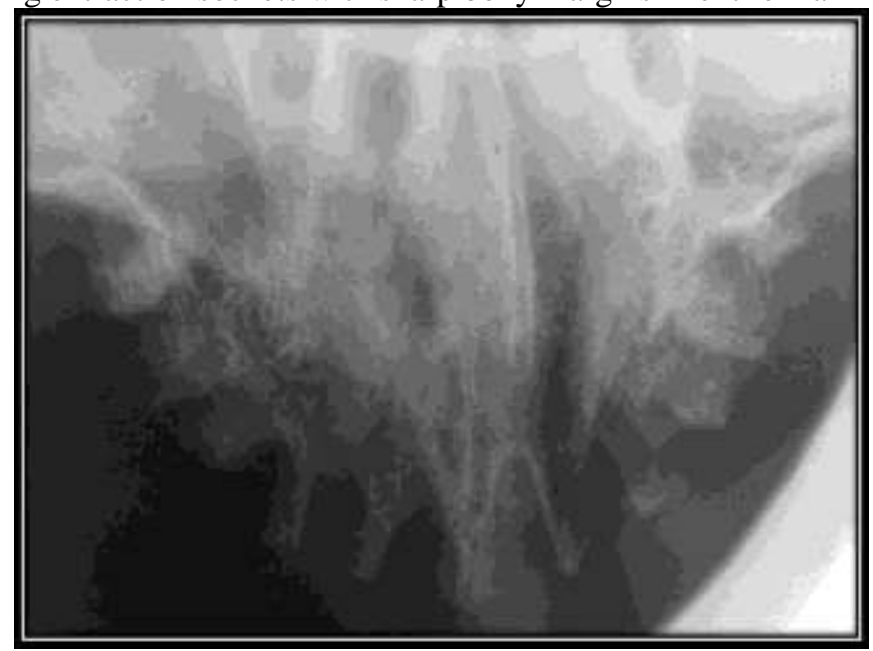

5. OPG showing extraction sockets with sharp margins. Floor of maxillary sinus is not traceable bilaterally. Enostosis noted in periapical region of 44 . 


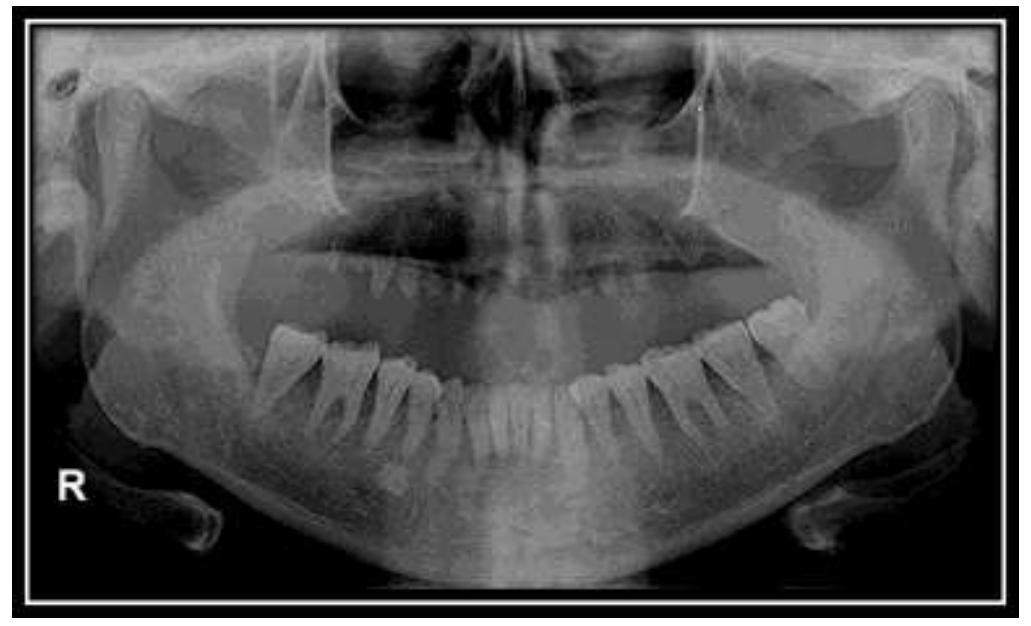

6. Water's view showing near total opacification of right and left maxillary sinus.

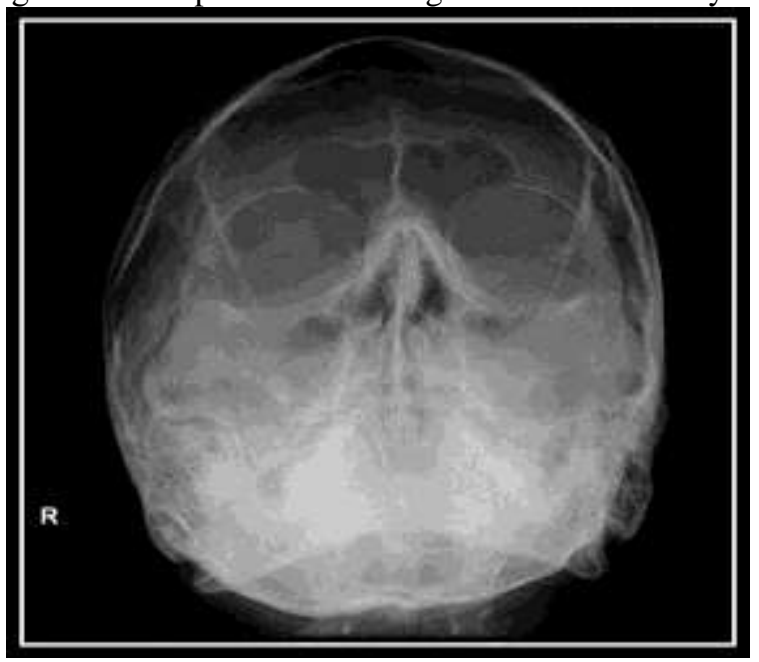

7. $\mathrm{CBCT}$ coronal view shows maxillary alveolus destruction with sinus involvement. White arrow shows sequestration and separation of maxillary alveolus from remaining maxilla. Red arrow shows opacification of ethmoid cells. Note the intact ostium on both sides.

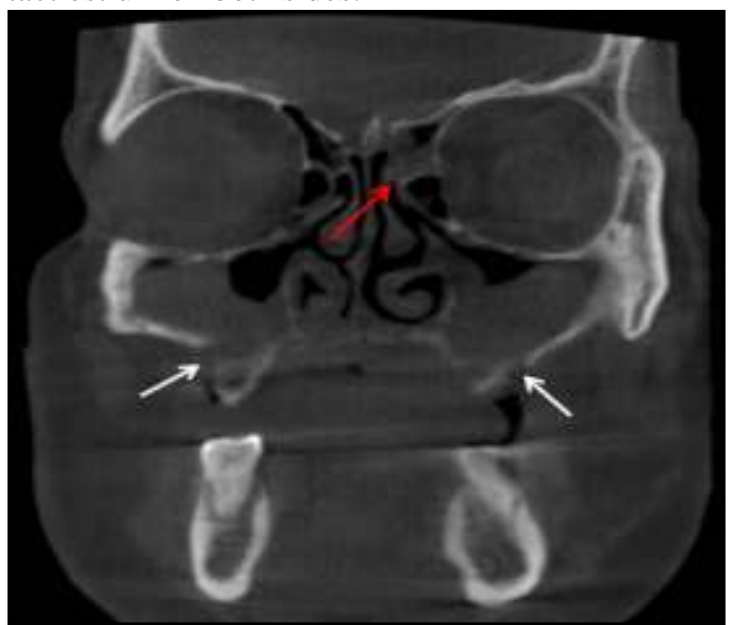

8. Axial view of CBCT showing irregular destruction of maxilla. Perforations of cortical plates noted bilaterally in entire maxillary alveolus. 


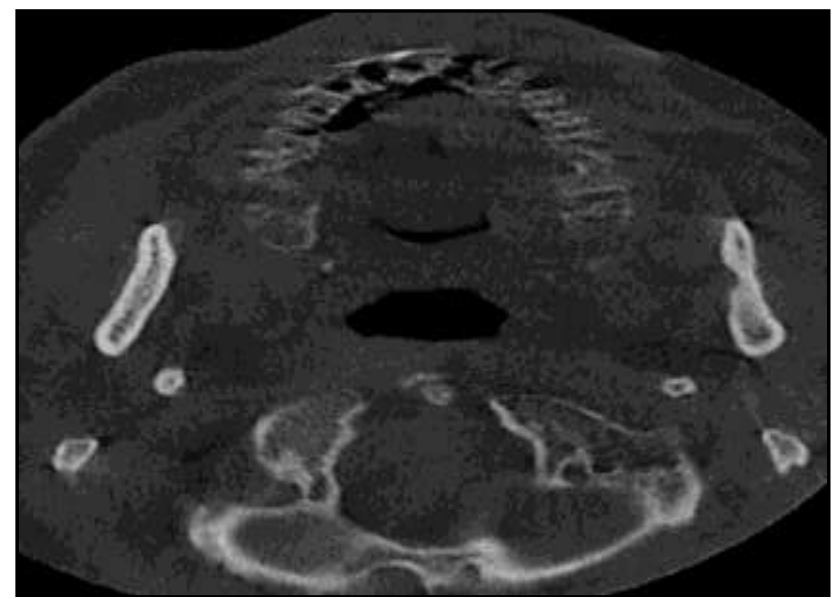

9. Axial view at higher section showing destruction of maxillary sinus walls.

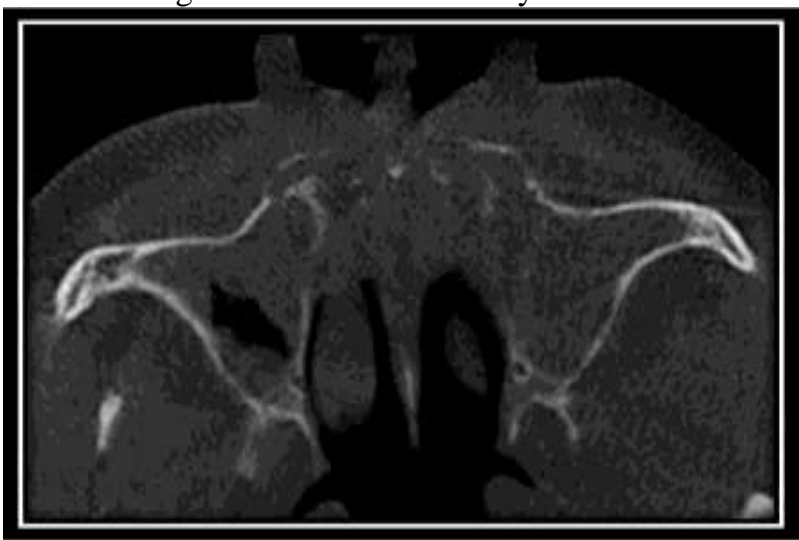

10. Sagittal section showing destruction of maxillary sinus floor with partial sinus opacification. Also note enostosis in mandible.

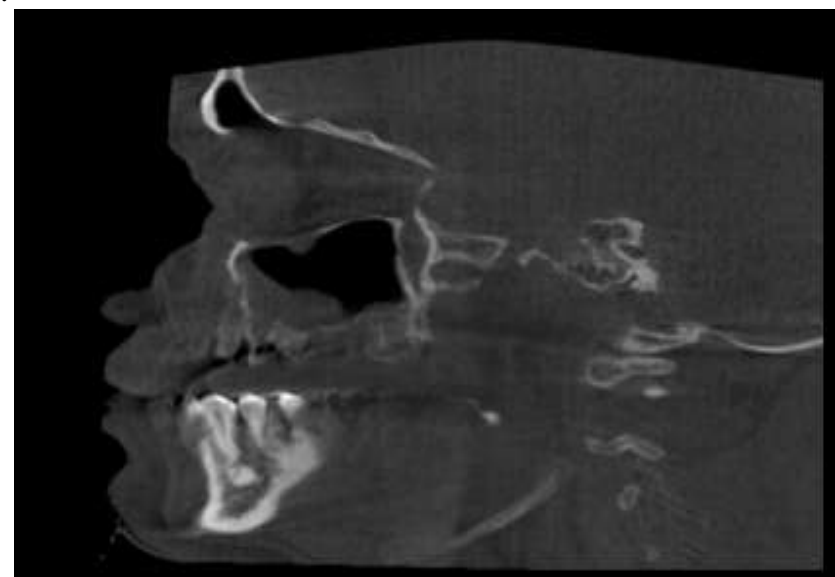

11. Sagittal section at different level. Red arrow indicates mucosal thickening of frontal and sphenoidal sinuses. White arrow shows intact spheno-ethmoidal recess. 


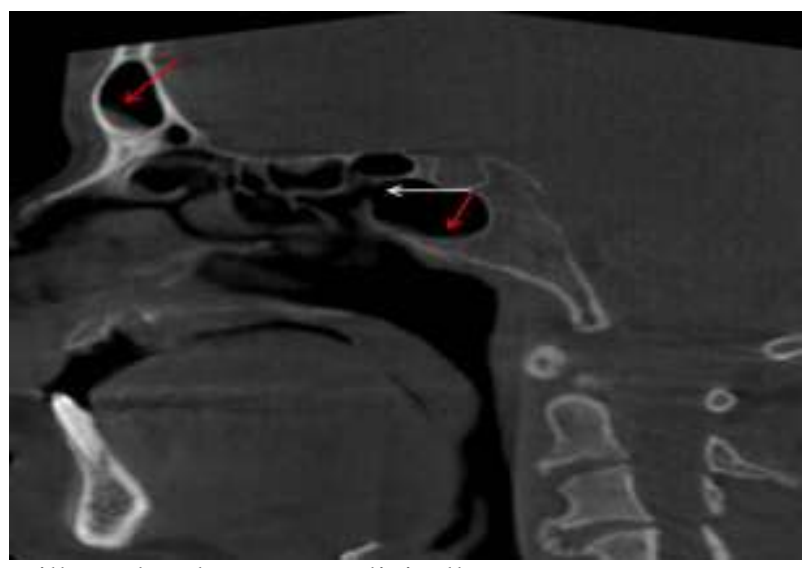

12. Postoperative healed maxillary alveolus as seen clinically.

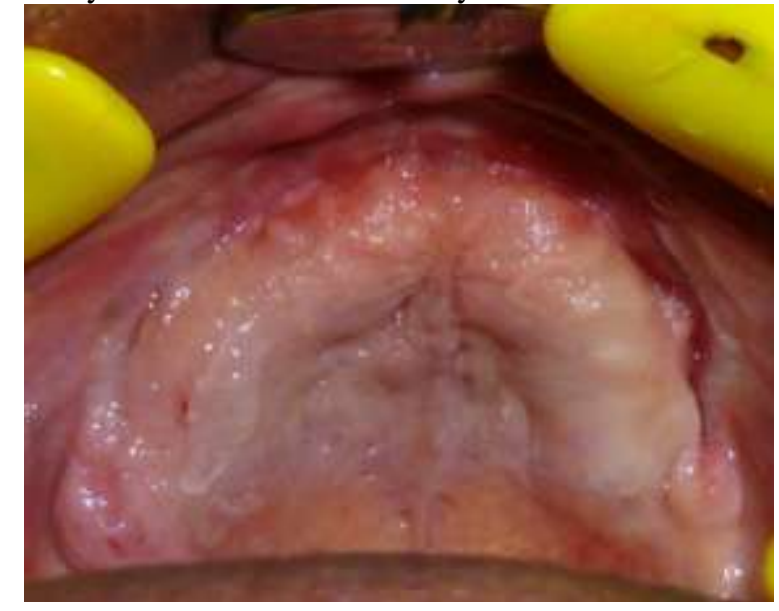

13. Radiographic picture (OPG) of postoperative healing. Note the intact floor of bilateral maxillary sinus.

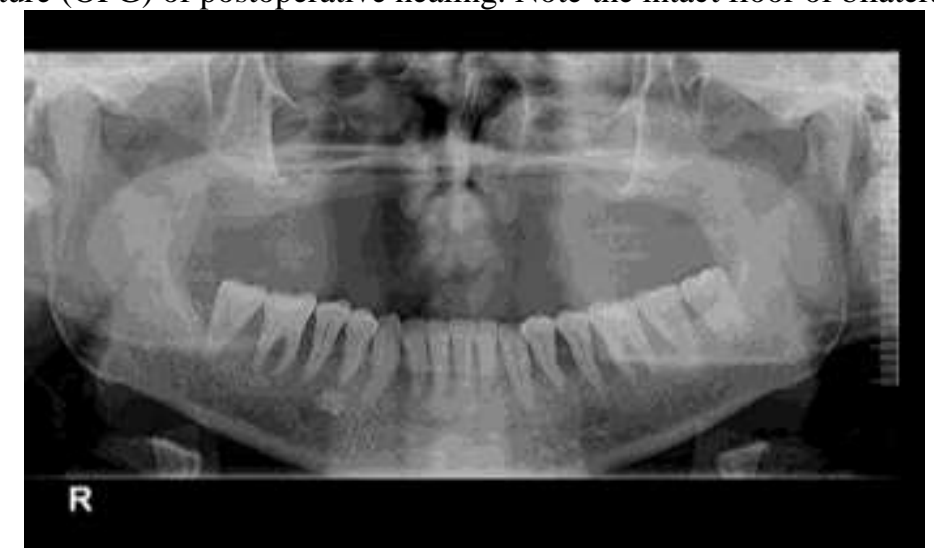

14. Clinical picture after delivery of complete denture.

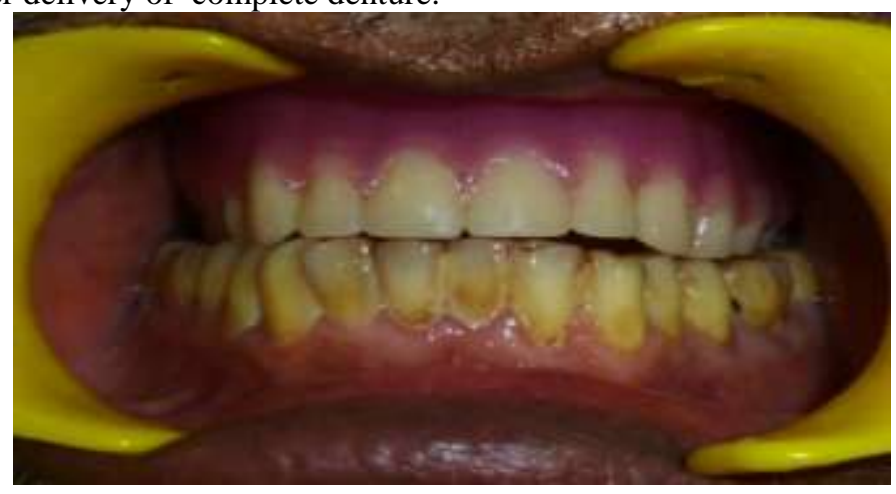

